\title{
Novel fabrication technique for planar glass waveguides
}

\author{
D W J Harwood, E R Taylor, C T A Brown, D P Shepherd, D N Payne
}

\begin{abstract}
:
A novel technique has been developed for the deposition of low-loss planar glass waveguides by directly spin coating from the liquid, thus overcoming the problems of reproducing glass stoichiometry when depositing from the vapour.
\end{abstract}

\section{Introduction}

Novel glasses, e.g. fluoroaluminates, chalcogenides, are important for future optical waveguide devices, such as $1.3 \mu \mathrm{m}$ planar amplifiers and non-linear switches. However, they are difficult to fabricate as thin films because of their tendency to crystallise and volatility. Reproducing the stoichiometry of multi-component glasses in planar waveguide form has traditionally proved difficult by conventional techniques, such as sputtering, due to the different vapour pressures of each element [1]. Pulsed laser deposition (PLD) can overcome this problem because of the high vaporisation rates provided by excitation at ultraviolet wavelengths, but is often troubled by poor surface topography [2]. By dissolving the bulk material in a solvent, spin or dip coating can be used to retain the waveguide composition for a limited range of materials such as sol-gel glasses [3].

At temperatures above the liquidus temperature, several soft glass families, such as the fluorides, sulphides and phosphates, have viscosities comparable to those of spin coating solutions. A neodymium-doped fluoroaluminate (AlF) glass, with a viscosity of 0.1 poise at $1000^{\circ} \mathrm{C}$, has successfully been spin coated into a waveguide geometry (Figure 1) directly from its liquid phase. Laser operation around 1050nm has been observed in a 20 micron thick, $0.5 \%$ Nd-doped glass waveguide fabricated using this technique.

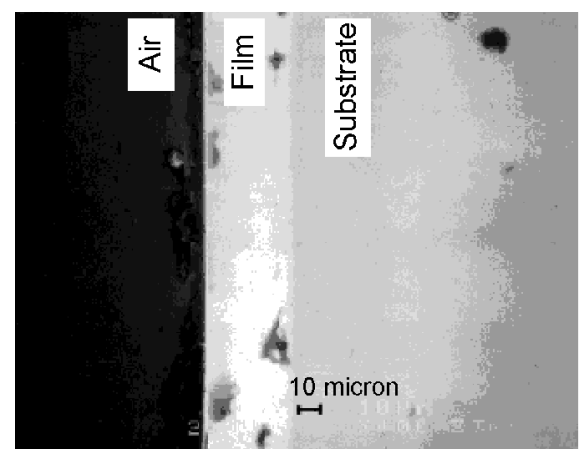

Figure 1: Cross-sectional SEM picture of a 40 micron thick AlF waveguide fabricated by spin coating
Performance is consistent with propagation losses of $0.5 \mathrm{~dB} / \mathrm{cm}$, which have been independently measured by imaging the scattered light from the waveguide surface.

\section{Fabrication of waveguides}

Nd-doped waveguides were fabricated using the apparatus shown in Figure 2. A $3 \mathrm{~mm}$ thick, 25mm diameter AlF glass substrate of refractive index 1.417 was preheated to its glass transition temperature of $430^{\circ} \mathrm{C}$ in an upper annealing furnace. In the lower furnace, a $30 \mathrm{~g}$ melt of $\mathrm{Nd}-$ doped $\mathrm{AlF}$ glass of refractive index 1.432 was heated to $1000^{\circ} \mathrm{C}$.

When the temperatures of the two furnaces had stabilised, an Aerotech ATS0200 positioning system was used to transfer the molten glass between furnaces. Accurate control of the melt position resulted in the surface of the substrate being dipped a fraction of a millimetre into the molten glass. The computer was used to synchronise the withdrawal and spinning of the substrate up to a maximum speed of 6000rpm. The as-deposited waveguides were then annealed in the upper furnace at $\mathrm{T}_{\mathrm{g}}=430^{\circ} \mathrm{C}$ for three hours, followed by a slow ramp down to room temperature at $30^{\circ} \mathrm{C} /$ hour.

\section{Characterisation of waveguides}

Scanning electron microscopy was used to examine the cross-section of the films. The waveguides exhibited excellent thickness uniformity $(0.1 \mu \mathrm{m} / \mathrm{mm})$ over the central area of the substrate, with some curvature of the surface observed at the edges due to re-melting of the underlying substrate glass. The high quenching rates achieved by coating a thin layer of molten glass onto a much larger cooled substrate produce a crystal-free glass/waveguide interface, as seen in SEM micrographs (Figure 1).

In order to measure the propagation losses, a $2 \mathrm{~mW}$ $\mathrm{HeNe}$ laser was end-coupled into a 20 micron thick 
parallel end-polished sample. A silicon CCD camera was then used to image the scattered radiation from the waveguide surface. An

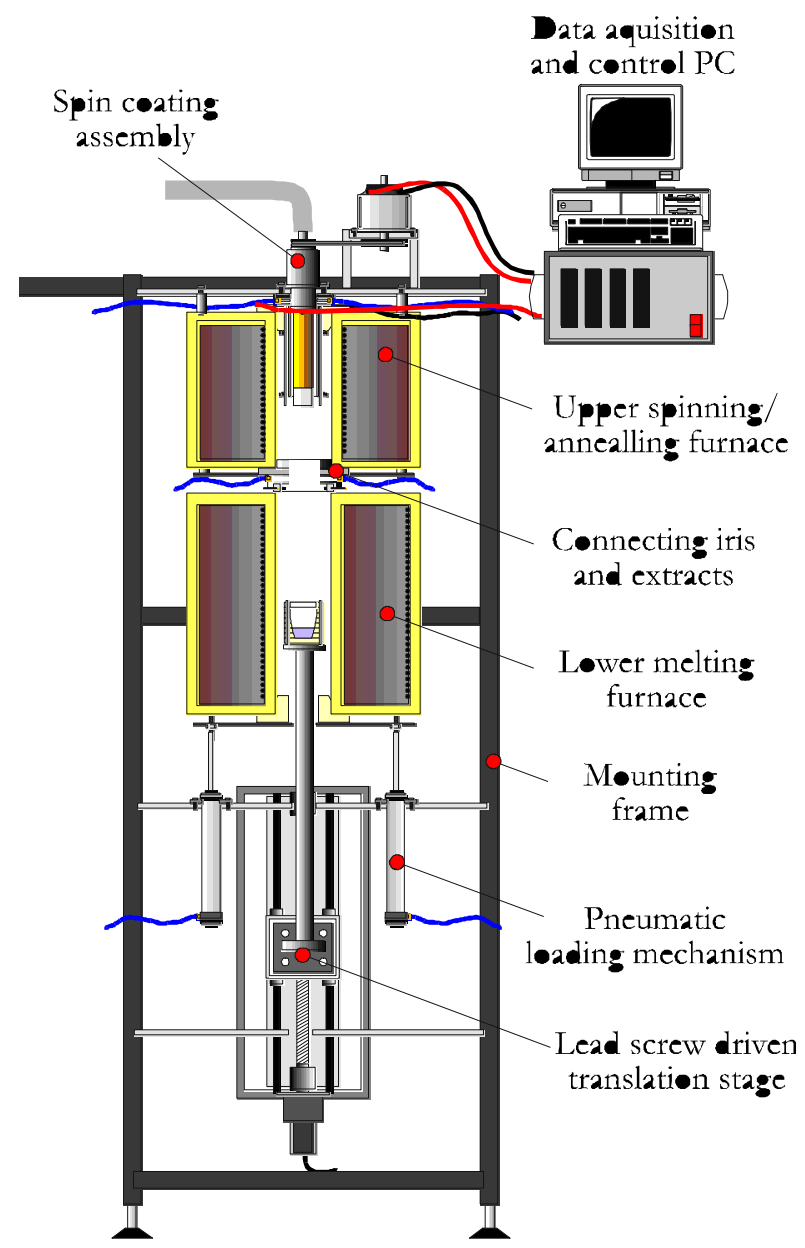

Figure 2: Apparatus for spin coating of waveguides using molten glass.

exponential fit to the scattered intensity along the length of the waveguide gave a loss of $\sim 0.5 \mathrm{~dB} / \mathrm{cm}$.

\section{Laser operation around 1050nm}

A laser cavity was formed by the attachment of plane mirrors to the polished, parallel end faces of a 20 micron thick, $5 \mathrm{~mm}$ long waveguide. The chopped output from a Ti:sapphire laser operating around $800 \mathrm{~nm}$ was end-launched into the waveguide using a $50 \mathrm{~mm}$ focal length microscope objective. The input coupler was highly reflecting (HR) at $1050 \mathrm{~nm}$, and highly transmitting (HT) at $800 \mathrm{~nm}$. A $3.5 \%$ output coupler at $1050 \mathrm{~nm}$ was used which was $\mathrm{HR}$ at $800 \mathrm{~nm}$ in order to double pass any unabsorbed pump.

Laser output was observed at several wavelengths between $1045 \mathrm{~nm}$ and $1065 \mathrm{~nm}$, as shown in Figure 3. The peaks correspond to the positions of maximum reflectivity in the Fabry-Perot cavity formed between the surface of the mirrors and the back of their respective substrates. The maximum slope efficiency was measured to be $10 \%$ with respect to incident power for a corresponding threshold of $170 \mathrm{~mW}$ (Figure 4).

The measured slope efficiency is consistent with the waveguide losses described earlier. These losses are similar to the typical bulk losses of the glass of $0.3 \mathrm{~dB} / \mathrm{cm}$ and can be expected to improve with higher-purity starting materials for the glass.

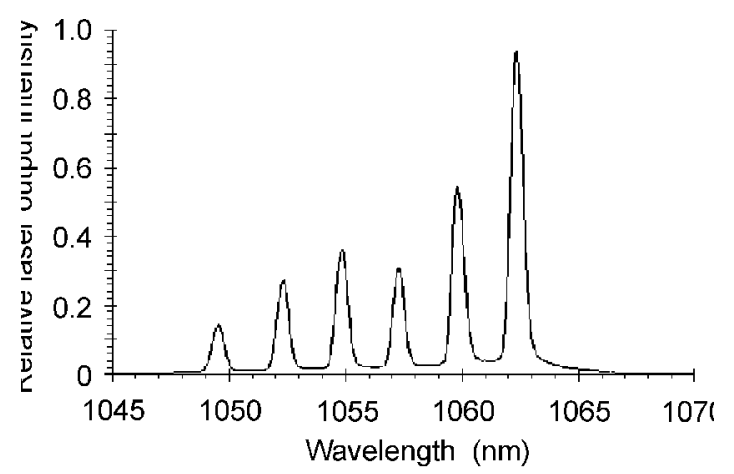

Figure 3: Lasing spectrum for an Nd-doped AlF glass waveguide illustrating operation on several lines.

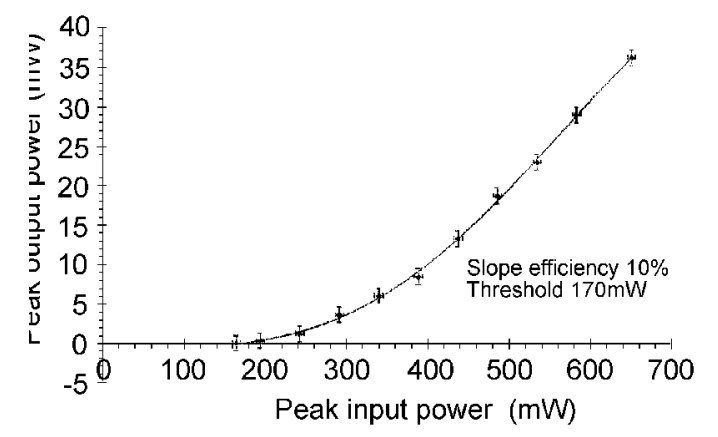

Figure 4: Output power at $1050 \mathrm{~nm}$ as a function of pump power at $800 \mathrm{~nm}$ for an $\mathrm{Nd}$ doped AIF glass waveguide laser.

\section{Conclusion}

Laser operation around 1050nm has been observed for the first time, to our knowledge, in a Nd-doped planar waveguide prepared by spin coating of a fluoroaluminate glass directly from the liquidus phase. Losses in the waveguide were determined to be of the order of $0.5 \mathrm{~dB} / \mathrm{cm}$ compared with typical bulk losses of $0.3 \mathrm{~dB} / \mathrm{cm}$. 


\section{Further work}

Future use of the technique will concentrate on the fabrication of overclad waveguides. A two-stage spin coating process will be applied to both bury the waveguides, for a reduction in the propagation losses, and to reflow the original guiding layer, thus reducing the guide dimensions to single mode.

The Nd-fluoroaluminate glass composition has been designed and optimized for use as a $1.3 \mu \mathrm{m}$ amplifier in the important second telecommunications window. Low loss waveguides fabricated using this technique will lead to the fabrication of a low-cost integrated amplifier for this region.

\section{Acknowledgements}

This work was funded by EPSRC with CASE support from GEC-Marconi Research.

\section{References}

[1] G.R.J.Robertson et al. APPLIED OPTICS, 1991, 30, 3, pp. 276-278

[2] C.N.Afonso et al. APPLIED SURFACE SCIENCE, 1996, 96-98, pp.760-763

[3] X.Orignac et al. APPLIED PHYSICS LETTERS, 1996, 69, 7, pp. 895-897 